\title{
Retraction note: Microstructure of Co Precipitation Strengthened B2-Ordered NiAl
}

\author{
Chang-Sup Oh, Chang-Suk Han, Chang-Hwan Chun, Kyung-Wan Koo, Jang-Woo Kim, Young-Woo Kim, \\ Seung-Oh Han, Chang-Hwan Bae, and Ju-Hee Lee
}

Retraction note: Met. Mater. Int., Vol. 16, No. 2, pp. 175-183 (2010)

DOI: $10.1007 / \mathbf{s} 12540-010-0404-1$

This Retraction Note expands on the Retraction Note previously published in Metals and Materials International, November 2014, Volume 20, Issue 6, p 1169 (http://dx.doi.org/10.1007/s12540-014-6024-4)

The editorial board of Metals and Materials International has decided to retract this article for reasons of plagiarism.

Several paragraphs and figures in the manuscript have been used without indication that those paragraphs and figures had been published previously. The most original source paper is:

Crystal structure and morphology of Co precipitates in B2-ordered (Ni,Co)Al

W. H. Tian, M. Hibino and M. Nemoto, Intermetallics 6 (1998) 121-129

The online version of the original article can be found under doi: 10.1007/s12540-010-0404-1

Chang-Sup Oh, Chang-Suk Han*, Chang-Hwan Chun, Kyung-Wan Koo, Jang-Woo Kim, Young-Woo Kim, Seung-Oh Han, Chang-Hwan Bae, and Ju-Hee Lee

Dept. of Defense Science \& Technology, Hoseo University,

Asan-si, Chungnam 336-795, Korea

e-mail: hancs@hoseo.edu

CKIM and Springer 